\title{
Analysis of $\mathrm{FOXO3}$ gene polymorphisms associated with human longevity.
}

\author{
Timothy A Donlon ${ }^{1,2}$, Philip MC Davy ${ }^{3}$, Bradley J Willcox ${ }^{1,4,{ }^{*}}$ \\ ${ }^{1}$ Department of Research, Honolulu Heart Program/Honolulu-Asia Aging Study (HAAS), Kuakini \\ Medical Center, Honolulu, Hawaii. \\ 2John A. Burns School of Medicine, University of Hawaii, Honolulu, Hawaii. \\ ${ }^{3}$ Institute for Biogenesis Research, University of Hawaii, Honolulu, HI, 96813, USA \\ ${ }^{4}$ Department of Geriatric Medicine, John A. Burns School of Medicine, University of Hawaii, \\ Honolulu, Hawaii.
}

\begin{abstract}
Next-generation DNA sequencing has ushered in a new era of genotype-phenotype comparisons that have the potential to elucidate the genetic nature of complex traits. Since such methods rely on short sequence reads and since the human genome is composed largely of repetitive DNA elements larger than these read lengths many results cannot be mapped and are discarded, thus eliminating a large portion of the genome from analysis. Discerning associations in complex traits, such as longevity, will require either longer read lengths or methods to address these sequence complexities. Whole genome analysis, such as Genome Wide Association Studies (GWAS) also suffers from the repetitive nature of the human genome, as there exist many gaps in the availability of useable genetic markers, often in interesting regulatory regions. Methods are described here whereby some of these problems have been addressed by targeted DNA sequencing, full exploitation of available public databases, and a careful evaluation of genomic features where we use the FOXO3 gene as an example to identify functional variations and how they may relate to longevity.
\end{abstract}

\section{Keywords}

long—range DNA sequencing; Long—range PCR; genome complexity; repetitive DNA

\section{Introduction}

About half of the human genome is comprised of repeats; composed of transposable elements, satellite DNAs, gene families, duplicated chromosomal regions, and pseudogenes $(1,2)$. The simple repeat family, "Alu element", is $~ 300$ bp in length and occupies about $10 \%$ of the genome. The problem of aligning these repeats is a recurrent difficulty in sequence mapping algorithms so that the automated detection of such elements is no trivial

*Corresponding authors: B. J. W. (willcoxbj@gmail.com). 
task. DNA repeats are filtered out by most DNA alignment programs (i.e. RepeatMasker and Censor). To date, the most commonly used NGS platforms provide 200-300 bp sequence reads that must be integrated into a reference genome. For de novo assembly, repeats that are longer than the read length create gaps in the assembly and are ignored. This fact, coupled with the short length of NGS (next-generation sequencing) output, results in a more fragmented assembly than Sanger sequencing, in which read lengths are $\geq 700 \mathrm{bp}$. In addition to creating gaps, repeats can be erroneously collapsed on top of one another and can cause complex, misassembled rearrangements. Almost 30 million base pairs (Mbp) of euchromatic genome sequence are either absent from, or have no assigned locations in, current assemblies of the human genome, presumably due to the limitations in mapping repetitive elements. (3) This problem has led to an abundance of single nucleotide polymorphisms (SNPs) in public databases that are from non-allelic, homologous sequences, such as pseudogenes and gene families. Take $F O X O 3$ and its pseudogene, $F O X O 3 B$, for example. As of August 8, 2011, there were 38 reported sequence variants in the FOXO3 coding region that included 21 synonymous, 16 non-synonymous (including our newly found variant), and 2 frame-shift variants (GRCh37, NCBI dbSNP Build 134). After chromosome 6-specific, long-range PCR amplification, sequencing, and genomic mapping these SNPs were found either to reside in the FOXO3B pseudogene on chromosome 17 or were misidentified by $F O X O 3-F O X O 3 B$ alignment (4). We were able to sequence a $120 \mathrm{~Kb}$ region of the FOXO3 gene in order to filter out all the erroneous SNPs so that we could develop a better understanding of the potential functional variants associated with longevity (5).

In this chapter, we describe the methods used to discriminate between true DNA sequence variants and the plethora of unfiltered data present in the public domain in order to provide guidelines for a more successful campaign of enumerating genotype-phenotype correlations for complex traits.

\section{Materials}

Prepare all solutions using ultrapure water (prepared by purifying deionized water, to attain a sensitivity of $18 \mathrm{M} \Omega-\mathrm{cm}$ at $25^{\circ} \mathrm{C}$ ) and analytical grade reagents. Prepare and store all reagents at room temperature (unless indicated otherwise). Diligently follow all waste disposal regulations when disposing waste materials. All equipment should be nuclease-free to reduce DNA degradation.

\subsection{Region-specific Amplification}

1. For long-range DNA amplification, primers should have a Tm ranging from 68 $70^{\circ} \mathrm{C}$. Check all primers against BLAST to make sure there are no two forward and reverse primers near one another and be sure to inactivate the "mask repeats" option when performing a BLAST screen so that a complete analysis of sequence homologies can be assessed.

2. Long-range DNA amplification may be performed using either a mixture of rTth and VENT® polymerases or a recombinant enzyme kit 
3. PCR product clean-up columns or 96-well filter plates can be used for isolating product from primers,

4. A 384-well format is also available for a larger number of samples,

5. Vacuum pump for filter plates used cleaning up PCR product or centrifuge that will hold a sufficient number of samples

6. Vacuum manifold for attaching plate,

7. Microtiter plate shaker for mixing.

\section{Methods}

\subsection{Long-range DNA Amplification}

A method of long-range PCR (6) can be used to generate locus-specific products of 16-18 $\mathrm{kb}$ in order to avoid complications from repetitive DNA (see Note 1).

\subsubsection{PCR Purification, Using a 96 Ultra-Filtration (UF) Plate on a vacuum manifold (see Note 2).}

1. Prepare the vacuum manifold according to the instructions. Place a waste tray inside the base of the manifold.

2. Place the appropriate UF Plate (selected for your PCR product) on top of the vacuum manifold.

3. Pipet the PCR samples onto the UF Plate (see Note 3).

4. Apply a vacuum and maintain at -800 mbar for 10 minutes or until the wells are completely dry. Slowly release pressure and switch off vacuum source (see Note $4)$.

5. (Optional Wash Step): Add $50 \mu \mathrm{l}$ deionized water to each well, apply a vacuum, and maintain at -800 mbar for 10 minutes or until the wells are completely dry. Switch off vacuum source. Note: The purity of the DNA obtained after elution is sufficient for most applications without this wash step being required. If a higher purity is needed for a specific application, this step should be carried out.

6. Carefully remove the UF Plate from the vacuum manifold.

7. Carefully tap the 96 UF Plate on a stack of clean absorbent paper to remove any liquid that might remain on the bottom of the plate.

8. Add $20 \mu \mathrm{l}$ deionized water to each well (see Note 5).

9. Shake the UF Plate on a microplate shaker for 2 minutes at medium speed.

Note: Ensure that the UF Plate is fixed securely on top of the shaker.

9a. Alternatively, purified DNA may be dissolved by pipetting samples up and down 20 times. 
10. Recover the purified PCR product by pipetting the eluate out of each well. For easier recovery of the eluates, the plate can be held at a slight angle.

\subsubsection{PCR Purification, Using the 96 UF Plate: Centrifugation Method}

1. Pre-wet membrane for maximum sample recovery.

2. Place a 96 filter plate over a solid-bottom receiver plate. Make certain that the receiver plate and the filter plate wells correspond to fit. Use a plastic tape to keep the two plates together.

3. Add $50 \mu \mathrm{L}$ of PCR dH2O into well of the UF filter plate.

4. Place filter and receiver plates together into a standard swinging bucket microtiter plate rotor assembly.

5. Centrifuge at $2,000 \times \mathrm{g}$ for 15 minutes.

6. After all fluid has evacuated from the well(s), discard receiver plate.

7. Place the pre-wetted filter plate over a new solid-bottom receiver plate. Use a plastic tape to keep the two plates together.

8. Add $25 \mu \mathrm{L}$ of PCR sample(s) into the pre-wetted filter plate well(s).

9. Place filter and receiver plates together into a standard swinging bucket microtiter plate rotor assembly.

10. Centrifuge at 2,000 to $3,000 \times \mathrm{g}$ for 10 minutes. (Centrifuge time may vary, depending upon concentration of sample (see note 6)

11. After the centrifugation, add $20 \mu \mathrm{L}$ of buffer $\left(\mathrm{TE}^{-4}\right)$ to each well. Pipette the buffer $\left(\mathrm{TE}^{-4}\right)$ up and down several times (10 to 20 time). Allow the plate to sit for 10 minutes, then aliquot the liquid into a new PCR plate.

12. Determine DNA concentration for DNA sequencing reactions.

\subsection{DNA Sequencing}

Primers can be designed using a wide variety of free on-line tools. It is imperative to remove unincorporated primers prior to sequencing. Amplified products are purified using a number of products, such as columns noted above or ethanol precipitation, and sequenced by either capillary electrophoresis or next-generation sequencing. (see note 7)

\subsection{DNA Sequence Alignment}

There's a plethora of DNA sequence alignment software, the input of which will depend on the sequencing methods output files. Large structural variation may be assessed by digesting the long-range PCR products with EcoRI and/or HindIII and comparing digests on a $0.7 \%$ agarose gel.

\subsection{Identification of Functional Variants}

There is a huge list of putative variants present in public databases, such as dbSNP. Many of these variants are merely sequencing errors and need to be filtered out before searching for 
functional variants. The 1,000 genomes project has reduced the number of error-derived variants. Actual variants can be identified by including those present in two or more subjects (Table I for example). For our FOXO3 project, all variants were screened on the RegulomeDB site, which includes known and predicted regulatory elements in the intergenic regions, as well as regions of DNAase I hypersensitivity, binding sites for transcription factors, and promoter regions that have been shown to regulate transcription. The strength of this site is that it highlights the location of the SNP relative to sites of chromatin modification and transcription factor binding and highlights the SNP relative to the TFBS canonical sequence so that it can be easily determined if the SNP would be expected to modify binding. One of the shortcomings of RegulomeDB is that it does not evaluate variants for their effects on chromatin modification and binding efficiencies, something that can be quite challenging. Sources of these data included public datasets from GEO, the ENCODE project, and published literature (7). Unless otherwise indicated, all locations on chromosome 6 use the GRCh37.p13 genome build (http://www.gencodegenes.org/releases/ 19.html).

We also screened the FOXO3 variants using HaploReg (http://archive.broadinstitute.org/ mammals/haploreg/haploreg.php), which is a tool for exploring annotations of the noncoding genome at variants on haplotype blocks, such as candidate regulatory SNPs at disease-associated loci (8). Using LD information from the 1000 Genomes Project, linked SNPs and small indels can be visualized along with chromatin state and protein binding annotation from the Roadmap Epigenomics and ENCODE projects, sequence conservation across mammals, the effect of SNPs on regulatory motifs, and the effect of SNPs on expression from QTL studies. We searched HaploReg version 4.1 for the region chr6:108866973-109011102 using the Nov 5, 2015 build, hg38. By selecting an individual SNP, one can obtain information on the tissue types that demonstrate differential chromatin modification, DNAse I sensitivity, and/or expression level. Select a SNP that has a changed motif and the page will reveal additional information. For example, at the bottom of the page is an alignment of the genomic sequence surrounding the SNP and the canonical sequences to the altered putative motifs. A score indicates the binding efficiencies (position weight matrix) of the reference and variant sequences (9). (see note 8)

\section{Notes}

1. When validating PCR primers via BLAST it is important to turn off the "mask repeats" function in order to determine the correct complementary sites available in the genome. Otherwise a primer containing a repeat may incorrectly be interpreted to be "unique". There will be many similar sequences over a long region of the large amplicon. Using primers with a higher annealing temperature will help to reduce the number of primer-complementary sequences in the large amplicon and provide cleaner results. Be aware of which enzyme you're using for PCR and whether it can survive an extended denaturation step, otherwise it will be necessary to perform a manual "hot start". The length of the target sequence will affect the required starting extension time; generally, allow 30-60 seconds per kb of target. 
2. A number of products are available for purifying PCR product from primers and are available in tube or plate format. The important parameters are a cut-off of about $50 \mathrm{bp}$. Be prepared to lose as much as half of the product in the clean-up phase.

3. Processing PCR sample volumes larger than $150 \mu \mathrm{l}$ may lead to increased processing time and incomplete primer removal.

4. If the PCR volume exceeds $50 \mu \mathrm{l}$, a longer vacuum time is needed. Apply the vacuum until all wells are dry. Approximately 10 minutes of vacuum application are needed for each $50 \mu \mathrm{l}$ PCR volume. DO NOT OVERDRY THE SAMPLES as they will be difficult to remove from the filter.

5. $\operatorname{DMSO}(50 \% \mathrm{v} / \mathrm{v}), 3 \times \mathrm{SSC}$, and $\mathrm{EB}(10 \mathrm{mM}$ Tris. $\mathrm{Cl}, \mathrm{pH} 8.5)$ or similar buffers can be used instead of water for elution.

6. Don't spin the plate too hard or the DNA may become dislodged and lost. Spin just hard and long enough to remove the excess liquid.

7. Again, select primers and screen them using BLAST (turn off "mask repeats").

DNA sequencing technologies are improving all the time. The important issue to keep in mind is that the genome is quite complex and any methods that can be used to reduce this complexity will result in a more complete evaluation of variation. As sequencing reads become longer (i.e. 500 bp) the need for locusspecific amplification is reduced since it will be more accurate to accurately map the most common repeats, such as Alu, which are $\sim 300$ bp in length.

8. Be aware of which genome assembly (i.e. build) for the SNPs you are searching or HaploReg will not return the correct region.

\section{References}

1. Schmid CW, Deininger PL (1979) Sequence organization of the human genome. Cell, 6(3): p. 345358

2. Batzer MA, Deininger PL (2002), Alu repeats and human genomic diversity. Nat Rev Genet. 3(5): p. 370-379 [PubMed: 11988762]

3. Lander ES (2011) Initial impact of the sequencing of the human genome. Nature 470: p. 187-197 [PubMed: 21307931]

4. Donlon TA, Curb JD, He Q, Grove JS, Masaki KH, Rodriguez B, Elliott A, Willcox DC, Willcox BJ (2012) FOXO3 Gene Variants and Human Aging: Coding Variants May Not Be Key Players. J Gerontol A Biol Sci Med Sci 67(11): p. 1132-1139 [PubMed: 22459618]

5. Donlon TA, Morris BJ, Chen R, Masaki KH, Allsopp RC, Willcox DC, Elliott A, Willcox BJ (2017) FOXO3 longevity interactome on chromosome 6. Aging Cell 6(5): p. 1016-1025

6. Cheng S1, Fockler C, Barnes WM, Higuchi R (1994) Effective amplification of long targets from cloned inserts and human genomic DNA. Proc Natl Acad Sci U S A 91(12): p. 5695-5699. [PubMed: 8202550]

7. Boyle AP, Hong EL, Hariharan M, Cheng Y, Schaub MA, Kasowski M, Karczewski KJ, Park J, Hitz BC, Weng S, Cherry JM, Snyder M (2012) Annotation of functional variation in personal genomes using RegulomeDB. Genome Research 22(9): p. 1790-1797 [PubMed: 22955989]

8. Ward LD, Kellis M (2012) HaploReg: a resource for exploring chromatin states, conservation, and regulatory motif alterations within sets of genetically linked variants. Nucleic Acids Res 40 (Database issue): p. D930-D934. [PubMed: 22064851] 
9. Kheradpour P, Kellis M (2013) Systematic discovery and characterization of regulatory motifs in ENCODE TF binding experiments. Nucleic Acids Research 42(15): p. 2976-2987 [PubMed: 24335146] 


\begin{tabular}{|c|c|c|c|c|}
\hline $\begin{array}{l}\text { Position Weight Matrix ID } \\
\text { (Library from Kheradpour and Kellis, 2013) }\end{array}$ & Strand & Ref & Alt & 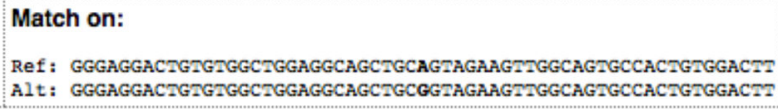 \\
\hline Ascl2 & - & 11.1 & 11.6 & NBYYVCASCIGCBVHNK \\
\hline HEN1_1 & + & 7.9 & 11.6 & DDDGGNMKCAGCTGCKBCCYHD \\
\hline HEN1_2 & + & 8.9 & 13.1 & KMKCAGCTGCKB \\
\hline Myf_1 & - & 17.1 & 6.6 & MRRCARCWGSWG \\
\hline Myf_3 & + & 13.5 & 12.5 & MNSVRVCAGGTGNHGDRN \\
\hline Rad21_disc8 & + & 11.4 & 11 & SSHGSSMNSwssws \\
\hline Znf143_disc3 & + & 12.3 & 11.4 & GSVBBSBGGGVVNBGBRGB \\
\hline
\end{tabular}

Figure 1. Example of Regulatory Motifs Altered by SNP (rs2802288) in HaploReg The online-database, HaploReg, was searched for SNP rs2802288. In addition to this SNP 12 other neighbors that are in linkage disequilibrium (LD) are shown on a new page and include information as the degree of LD, the population allele frequencies, histone marks, eQTL, proteins bound, and transcription factor/enhancer motifs that are changed by the SNP (not shown here). Selection of rs2802288 results in a list including histone marks and the cell types evaluated as well as a table of motifs that are altered by the SNP (shown above). This table shows the transcription factors, the strand \pm , reference sequence, SNP (Alt) sequence, and the canonical sequence for the motif (shown as a nucleotide ambiguity code). Selection of the following link: Position Weight Matrix ID (Library from Kheradpour and Kellis, 2013) will bring up a page that includes all the known canonical sequences for that transcription factor/enhancer so that they may be evaluated further. For example, RAD21_disc8 leads to a column whereby RAD21 may be selected to show various experiments that discovered RAD21 binding and their associated heatmap scores. Note that not all enhancers are present in the Weight Matrix ID and may require searching other databases, such as JASPAR (http://jaspar.genereg.net). 
Table I.

Relative Number of SNPs in Public Databases and the KHHP Cohort

\begin{tabular}{lllll}
\hline dbSNP (total) & dbSNA (MAF $\searrow$ ע.01) & HaploReg & RegulomeDB & KHHP Cohort \\
\hline 12,456 & 1,182 & 1,617 & 476 & 146 \\
\hline
\end{tabular}

The above information shows the number of single nucleotide polymorphisms (SNPs) from the on-line database "dbSNP" (total), including copy number variants with minor allele frequencies $\searrow 0.01$, HaploReg, RegulomeDB, , and the Kuakini Honolulu Heart Program cohort of 95 individuals $\geq 95$ years of age. 